\title{
Evaluation of Ketamine Hydrochloride in Combination with Midazolam, Dexmedetomidine and Butorphanol as Balanced Anaesthesia in Cats
}

Debajyoti Pal, Basanta Saikia, Kalyan Sarma, Bedanga Konwar, M.C. Lallinchhunga, J.K. Choudhary, Rahul Singh Arya

10.18805/IJAR.B-4786

\begin{abstract}
Background: As cats undergo stress at the time of presentation before surgery and therefore sedation is required often. It is very difficult to choose a balanced anaesthetic protocol during the surgical intervention in the case of a cat. Therefore, the present study was undertaken to evaluate the effect of ketamine hydrochloride in combination with midazolam, dexmedetomidine and butorphanol as balanced anaesthesia on clinico-physiological, haematological, serum biochemical profile in the surgical management of cats undergoing neutering.

Methods: The study was performed on 18 clinical cases of cats which were brought to the Teaching Veterinary Clinical Complex, College of Veterinary Sciences and Animal Husbandry (CVSc and AH), Central Agricultural University (CAU), Selesih, Aizawl, Mizoram for elective surgery such as neutering. Cats were randomly divided into 3 equal groups, i.e. Gr. A, Gr. B and Gr. C. Animals were premedicated with glycopyrrolate @ $0.01 \mathrm{mg} / \mathrm{kg}$, i/m. After 10 minutes of premedication, in Gr. A ketamine hydrochloride @15 mg/kg and midazolam @ $0.5 \mathrm{mg} / \mathrm{kg}$, in Gr. B ketamine hydrochloride@10 mg/ kg and dexmedetomidine@ $5 \mathrm{mcg} / \mathrm{kg}$ and in Gr. C ketamine hydrochloride@ $5 \mathrm{mg} / \mathrm{kg}$, dexmedetomidine@ $5 \mathrm{mcg} / \mathrm{kg}$ and butorphanol @0.2 mg/kg was administered intramuscularly. Clinicophysiological and haemato-biochemical profiles were evaluated at 0 minute (baseline), then at 15, 30 and 60 minutes after administration of anaesthetic agents to evaluate their anaesthetic effect.

Result: In the case of time for induction, the quality of induction, assessment of peri-operative analgesia, depth of anaesthesia and quality of recovery there was no significant difference among all three groups. In Gr. B, animals showed significantly higher duration of recumbency (DOE) and recovery. The assessment of peri-operative analgesic effect among the groups revealed that analgesia during the perioperative period appeared best in Gr. C protocol. In regards to depth of anaesthesia, ketamine hydrochloride in combination with dexmedetomidine and butorphanol group showed a better result. Rectal temperature decreased significantly $(P>0.05)$ up to $60^{\text {th }}$ minutes in $\mathrm{Gr}$. A, but it remained within the physiological range. Heart rate raised significantly $(p<0.05)$ from the baseline ( 0 minutes) onwards and decreased significantly $(p<0.05)$ till the end $(60$ minutes $)$ of the study in $\mathrm{Gr}$. C. The respiration rate was significantly $(p<0.01)$ different in between time intervals for all three groups. No significant difference was observed on haemato-biochemical observation except glucose level which significantly increased after induction of anaesthesia. In conclusion, treatment with ketamine hydrochloride in combination with dexmedetomidine and butorphanol led to acceptable sedation and mild changes in clinico-physiological, haematological, serum biochemical profile in comparison to the other two groups.
\end{abstract}

Key words: Butorphanol, Dexmedetomidine, Feline, Glycopyrrolate, Ketamine hydrochloride, Midazolam, Neutering.

\section{INTRODUCTION}

With the advancement in anaesthesia in veterinary sciences, balanced anaesthesia is one of the budding procedures among cats. As cats undergo stress at the time of presentation before surgery and therefore sedation is required often (Moffat, 2008). Use of many combinations of sedative drugs include midazolam, ketamine, butorphanol and an alpha-2 agonist, dexmedetomidine for feline sedation have been reported (Biermann et al., 2012).

Midazolam administered intramuscular (IM) injection alone can cause ataxia and dysphoria in cats, thus making restraint and handling more difficult (Ilkiw et al., 1996). Butorphanol is a synthetic morphine derivative with agonistic effects at the K-opioid receptors and partial agonist and antagonist activity at the $\mu$-opioid receptors and in cats it
Department of Veterinary Surgery and Radiology, Central Agricultural University, Selesih, Aizawl-796 014, Mizoram, India.

Corresponding Author: Debajyoti Pal, Department of Veterinary Surgery and Radiology, Central Agricultural University, Selesih, Aizawl-796 014, Mizoram, India.

Email: drdebajyotipalvetz@gmail.com

How to cite this article: Pal, D., Saikia, B., Sarma, K., Konwar, B., Lallinchhunga, M.C., Choudhary, J.K. and Arya, R.S. (2022). Evaluation of Ketamine Hydrochloride in Combination with Midazolam, Dexmedetomidine and Butorphanol as Balanced Anaesthesia in Cats. Indian Journal of Animal Research. DOI: $10.18805 /$ IJAR.B-4786.

Submitted: 23-09-2021 Accepted: 16-12-2021 Online: 14-01-2022 
Evaluation of Ketamine Hydrochloride in Combination with Midazolam, Dexmedetomidine and Butorphanol as Balanced....

provides visceral analgesia and leads to mild sedation when administered alone (Ansah et al., 2002). Ketamine is absorbed rapidly after IM administration, has a short onset of action and produces a dissociative state including analgesia and amnesia (Kohrs and Durieux, 1998). Dexmedetomidine, an alpha2- adrenergic, induces dosedependent sedation, analgesia and muscle relaxation in cats with marked decreases in heart rate, respiratory rate, temperature, cardiac output and transient changes in blood pressure (Granholm et al., 2006).

Many intramuscular drug combinations for sedation and short term anaesthesia have been investigated in cats previously but, to our knowledge, the effects on clinicophysiological, haematological, serum biochemical profiles in the surgical management of cats have not been thoroughly evaluated. This study aimed to evaluate the effect of ketamine hydrochloride in combination with midazolam, dexmedetomidine and butorphanol as balanced anaesthesia on clinico-physiological, haematological, serum biochemical profile in the surgical management of cats.

\section{MATERIALS AND METHODS Place of work}

College of Veterinary Sciences and Animal Husbandry, Central Agricultural University, Selesih, Aizawl, Mizoram.

\section{Year of experiment}

2 years.

\section{Age group and sex}

5 months- 2 years of both sexes.

\section{Animal}

The study was performed on 18 clinical cases of cats that were brought to the Teaching Veterinary Clinical Complex, College of Veterinary Sciences and Animal Husbandry, Central Agricultural University (CAU), Selesih, Aizawl, Mizoram for elective surgery. All cats were considered healthy based on clinical examination, haemato-biochemical evaluation before surgery and fasted overnight along with withholding of water for 6 hours, prior anaesthesia.

\section{Experimental deigned}

All the 18 cats were randomly divided into 3 equal groups and sedated as follows.
The chronological order of the experimental procedures is given in Fig 1.

CPP-Clinico-physiological parameters, $\mathrm{H}$ - haematological parameters, B-Biochemical Parameters IN- 10 minutes interval in between premedication and induction.

\section{Parameters study \\ Clinico-physiological parameters}

For clinical parameters following methodology were used: Table 1.

Physiological parameters such as heart rate (HRbeats/min), respiratory rate (RR-breaths/min) and rectal temperature (RT- ${ }^{\circ} \mathrm{C}$ ) were also recorded at same time intervals with standard procedure.

\section{Blood sampling and analysis}

$5 \mathrm{ml}$ blood was collected from cephalic or saphenous vein in sterile vials containing dipotassium ethylene diamine tetraacetic acid $\left(K_{2}-E D T A\right)$ and clot-activator vials at 0 minutes thereafter at 15,30 and 60 minutes of post-injection of anaesthetics. The haematological parameters viz. $\mathrm{Hb}$, PCV, TEC and TLC were analysed with a blood cell counter (MS4e, Franc) and biochemical parameters viz. blood glucose, gamma-glutamyl transferase (GGT), blood urea nitrogen (BUN) and creatinine were estimated by Evolution 201 UV-visible Spectrophotometer and Fuji drichem. Nx 500.

\section{Statistical analysis}

Statistical analysis was carried out by Statistical Package for the Social Sciences (SPSS) version 27.0. One way analysis of variance (ANOVA) was used for comparing different groups at different time intervals and paired sample t-test was used to compare different time intervals in the same group. Results were presented as mean \pm standard error (mean \pm SE) and differences were considered statistically significant when $\mathrm{P}<0.05$.

\section{RESULTS AND DISCUSSION \\ Evaluation of clinico-physiological parameters}

The effects of ketamine hydrochloride in combination with midazolam, dexmedetomidine and butorphanol on clinical parameters are depicted in Fig 2.

In the case of time for induction, there was no significant difference among all three groups. In case of induction time, $\mathrm{Gr}$. B (9.5 \pm 2.0 minutes) and $\mathrm{Gr} . \mathrm{C}(10.5 \pm 1.7$ minutes) took

\begin{tabular}{|c|c|c|}
\hline Group $(n=6)$ & Premedicated & Anaesthetic treatment \\
\hline $\bar{A}$ & $\begin{array}{l}\text { Glycopyrrolate @ } \\
0.01 \text { mg/ kg, } \\
\text { intramuscularly, }\end{array}$ & $\begin{array}{l}\text { Ketamine hydrochloride @15 mg/kg and midazolam @0.5 mg/kg, } \\
\text { intramuscularly. }\end{array}$ \\
\hline B & $\begin{array}{l}10 \text { minutes } \\
\text { prior to }\end{array}$ & $\begin{array}{l}\text { Ketamine hydrochloride @10 mg/kg and dexmedetomidine@ } 5 \text { mcg/ } \\
\text { kg, intramuscularly. }\end{array}$ \\
\hline C & induction. & $\begin{array}{l}\text { Ketamine hydrochloride @ } 5 \text { mg/kg, dexmedetomidine@ } 5 \text { mcg/kg } \\
\text { and butorphanol@ } 0.2 \mathrm{mg} / \mathrm{kg} \text {, intramuscularly. }\end{array}$ \\
\hline
\end{tabular}


Evaluation of Ketamine Hydrochloride in Combination with Midazolam, Dexmedetomidine and Butorphanol as Balanced....

less time as compared to Gr.A (12.3 \pm 1.1$)$. It might be due to the action of dexmedetomidine as it is a potent $\alpha_{2}$ - adrenergic agonist leading to less duration of time for induction (Mantz et al., 2011). Duration of recumbency (DOR) and time for recovery were significantly $(p<0.01)$ more in the group- $B$
(134.3 \pm 4.1 minutes and $226.5 \pm 6.5$ minutes respectively) as compared to the $\mathrm{Gr}$. A $(37.1 \pm 1.6$ minutes and $115.3 \pm 4.2$ minutes respectively) and $\mathrm{Gr}$. C (48.8 \pm 6.0 and $123.0 \pm 20.1$ minutes respectively). This might be because of the synergistic effect of ketamine and dexmedetomidine in the

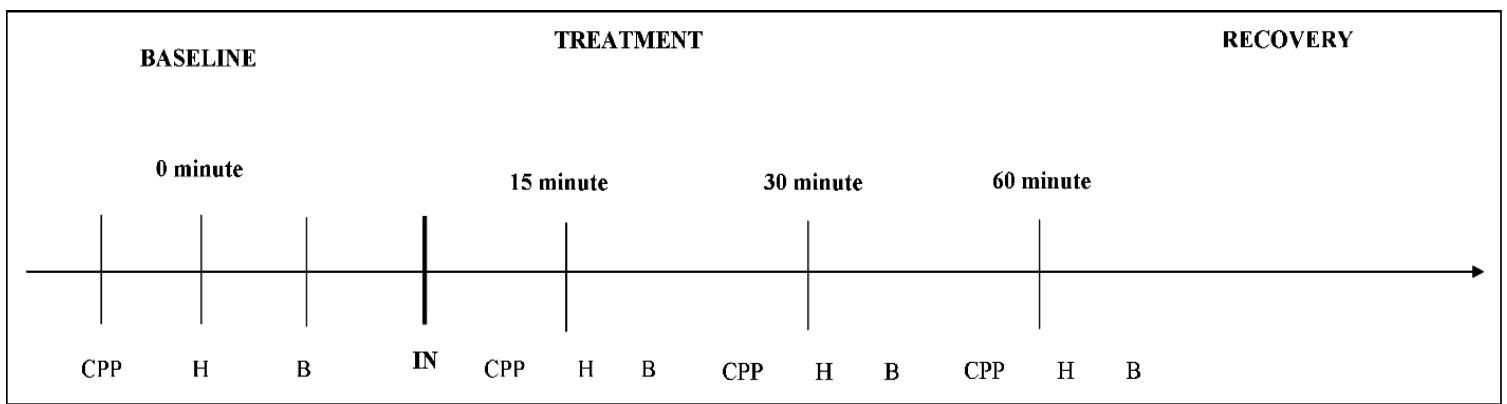

Fig 1: Chronological order of procedures of the experiments carried out as a cross-over study in three groups.

Table 1: Different parameters study under clinical parameters.

\begin{tabular}{|c|c|}
\hline Clinical parameters & Methodology \\
\hline Time for induction & $\begin{array}{l}\text { Time is taken in minutes from the administration of the anaesthetic agent to the loss } \\
\text { of reflex of the animal. }\end{array}$ \\
\hline Quality of induction & Scoring method described by Maddern et al. (2010) \\
\hline Assessment of perioperative analgesia & Scoring method described by Amengual et al. (2013) \\
\hline Depth of anaesthesia & Method described by Ahmad et al. (2013) \\
\hline Duration of recumbency & Time interval (in minutes) from induction to the cat's assumption of sternal posture. \\
\hline Time for recovery & Time interval (in minutes) between the injection of anaesthetic and the cat's ability to stand. \\
\hline Quality of recovery & As per the method described by Shah et al. (2019) \\
\hline $\begin{array}{l}\text { Assessment of post- operative analgesia } \\
\text { upto } 1 \text { hours (at every } 30 \text { minutes interval) }\end{array}$ & VAS described by Brondani et al. (2011) \\
\hline
\end{tabular}

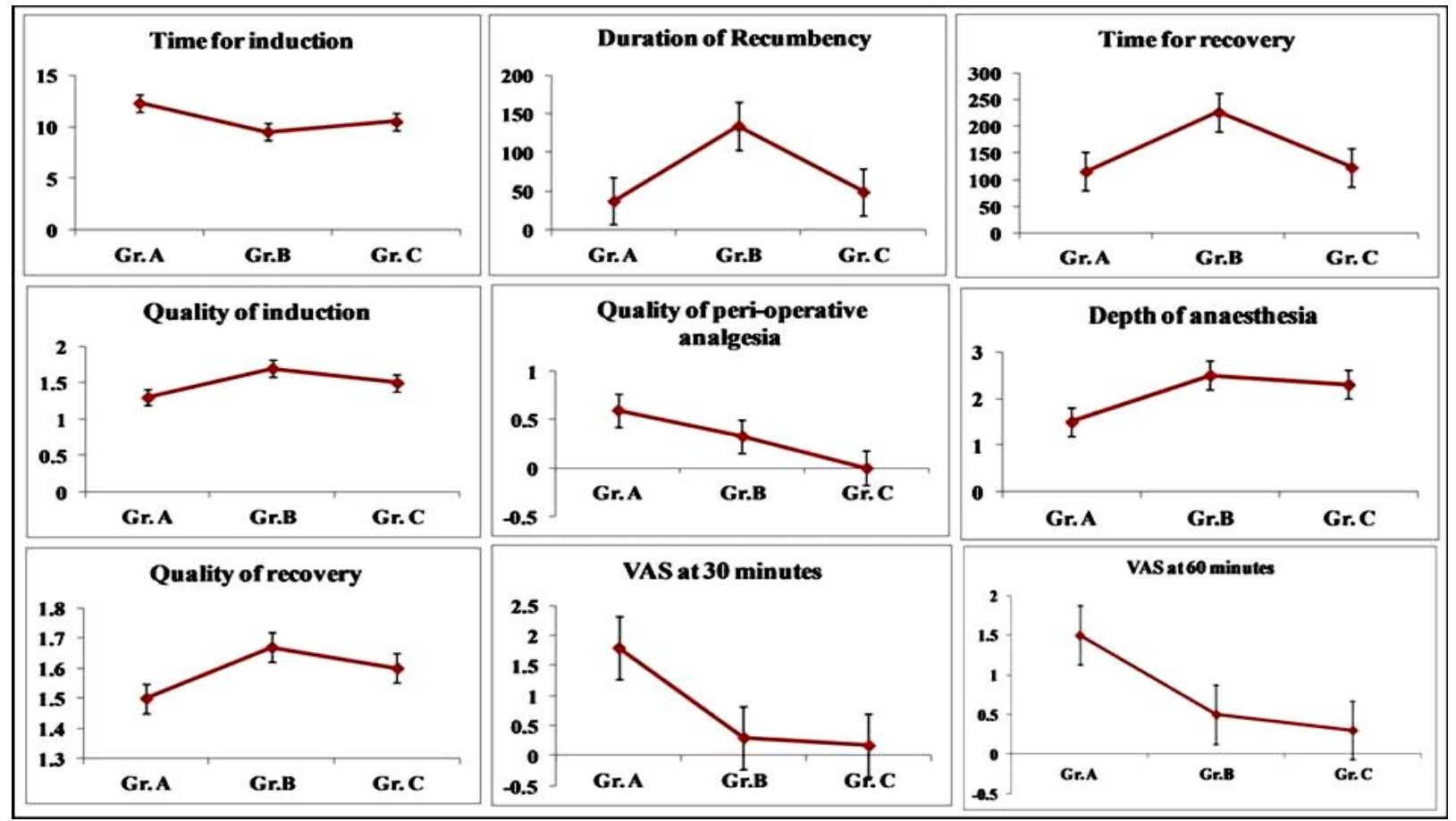

Fig 2: Effects of ketamine hydrochloride in combination with midazolam, dexmedetomidine and butorphanol on clinical parameters. 
animals and also because of the residual effect of ketamine (Selmi et al., 2003) and also the anxiolytics effect of the drug (Mantz et al., 2011).

There was no significant difference was observed in regards to the quality of induction, assessment of perioperative analgesia, depth of anaesthesia and quality of recovery among the groups. But, a better quality of induction

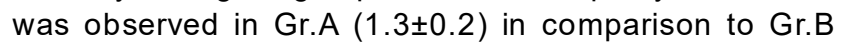
$(1.7 \pm 0.1)$ and Gr.C (1.5 \pm 0.2$)$. This might be due to the muscle relaxation property of midazolam and improves the reduction of anxiety along with good sedation Olkkola and Ahonen (2008). The assessment of perioperative analgesic effect among the groups revealed that analgesia during the perioperative period appeared best in group- $C(0.0 \pm 0.0)$ followed by Gr.B $(0.33 \pm 0.2)$ then $\mathrm{Gr} . \mathrm{A}(0.6 \pm 0.2)$. This was because of the opioid butorphanol present in Gr.C. Butorphanol is a potent opioid that diminishes the pain during the operative period Roy (2008). In regards to depth of anaesthesia, Gr.B $(2.5 \pm 0.2)$ showed better results than the other two groups $A$ and $B(1.5 \pm 0.2$ and $2.3 \pm 0.3$ respectively) which might be due to the suppress the $\alpha_{2}$ adreno receptors by dexmedetomidine, causing marked central nervous system depression (Mantz et al., 2011).

The assessment of postoperative analgesia (VAS) revealed significantly $(p<0.01)$ higher in the group- $A$ at 30 minutes and nonsignificant at 60 minutes $(1.8 \pm 0.4$ and $1.5 \pm 0.5$ respectively) as compared to the Gr.B and Gr.C $(0.3 \pm 0.2$ and $0.5 \pm 0.2$ and $0.17 \pm 0.2$ and $0.3 \pm 0.2$ respectively). The reason behind this might be because of the less analgesic effect of midazolam (Pasin et al., 2015) used in this group compared to the other drugs i. e. dexmedetomidine and opioid butorphanol.

The critical analysis revealed that ketamine hydrochloride in combination with dexmedetomidine and butorphanol (Gr.C) showed a good response on clinical parameters in pre and post-operative surgical management of cats.

\section{Physiological parameters}

The effect of anaesthesia on physiological parameters viz. rectal temperature, heart rate (beats/min) and respiratory rate (breaths/min) are shown in Fig 3.

Rectal temperature decreased significantly $(P>0.05)$ up to $60^{\text {th }}$ minutes in $\mathrm{Gr}$.A but it remained within the physiological range. The difference in rectal temperature during anaesthesia was insignificant among the three groups up to 30 minutes but a significant difference in rectal temperature was observed among three groups at 60 minutes (Fig 3). There was no significant difference $(p>0.05)$ in heart rates in Gr.A and Gr.C at different time intervals, except in $\mathrm{Gr}$. B. In Gr.B, heart rate raised significantly $(p<0.05)$ from the baseline (0 minutes) onwards and decreased significantly $(p<0.05)$ till the end $(60$ minutes $)$ of the study. The respiration rate was significantly $(p<0.01)$ different in between time intervals for all three groups. In all the groups, the respiratory rate significantly $(p<0.01)$ decreased starting from the baseline at 0 minutes and significantly raised $(p<0.01)$ towards the end of the study at 60 minutes. The respiratory rate was significantly $(p<0.05)$ decreased in Gr. B and Gr.C at 30 minutes and 60 minutes as compared to Gr.A (Fig 3).

The decrease in temperature might be because of peripheral vasodilation, reduced muscular and physiological activities, decreased metabolism. Heart rates were increased more in $\mathrm{Gr}$.A as compare to the other groups after administration of glycopyrrolate as the drug has anticholinergic effects and causes an increase in heart rates (Dyson and Davies, 1999; Jacobson et al., 1994). The decreased respiratory rates might be due to the effect of agents used, such as ketamine and dexmedetomidine, as it causes a certain level of decrease in respiratory rate (Mantz et al., 2011).

\section{Haematological parameters}

The study showed that there was a significant $(p<0.05)$ difference in $\mathrm{Hb}, \mathrm{PCV}$ and TEC among the different time intervals in all the three groups throughout the observation period (Fig 4). The baseline values of $\mathrm{Hb}, \mathrm{PCV}$ and TEC were significantly $(p<0.05)$ decreased upto 30 minutes posttreatment and raised towards the baseline value at 60 minutes intervals in all the groups. This trend might be due to the pooling of the circulating blood cells in the spleen or other vascular reservoirs due to the decreased sympathetic activity during sedation or anaesthesia.

There was a non- significant decrease in total leucocyte count in all the groups, this might be because of the sequestration of the spleen during anaesthesia. It is an indicator of trauma, pain or infection (Shekhawat, 2018).

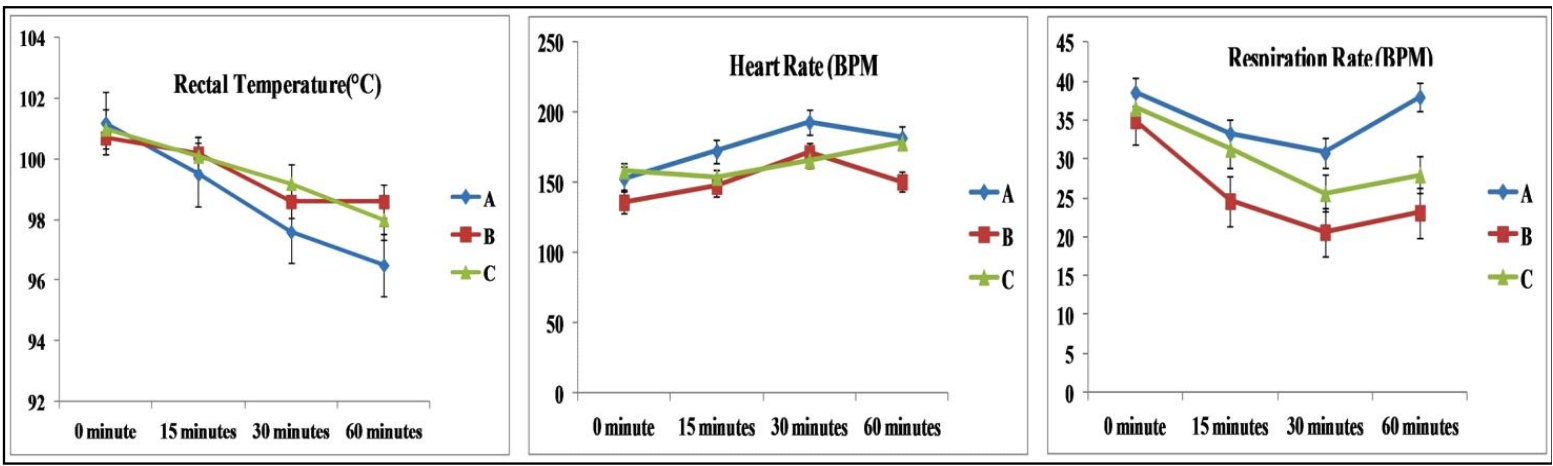

Fig 3: Effects of ketamine hydrochloride in combination with midazolam, dexmedetomidine and butorphanol on physiological parameters. 


\section{Biochemical parameters}

The anaesthetic effect of the drugs on biochemical parameters showed a significant $(P \leq 0.01)$ increase in glucose level in all the three groups in between different time intervals and the values were increased from the normal base level ( 0 minutes) towards 60 minutes till the end of the study (Fig 5). The reason behind hyperglycemia might be because of the anaesthetic drugs effect on the subcortical pathway, leading to stress with increased glucocorticoids, further mobilizing of glucose from the tissues to the circulation (Kelawala et al., 1991; David, 1993). There were no changes in the GGT value at different time intervals (Fig 5). It indicated that there was no adverse effect of the drugs used in this study on the liver. In all the groups, BUN values fluctuated within normal physiological range throughout the study. The effects seen might be due to the agents in the study (Shinde et al., 2018). The treatments did not affect creatinine in a different time interval. In all the groups of animals, the creatinine values were within the normal physiologic range.

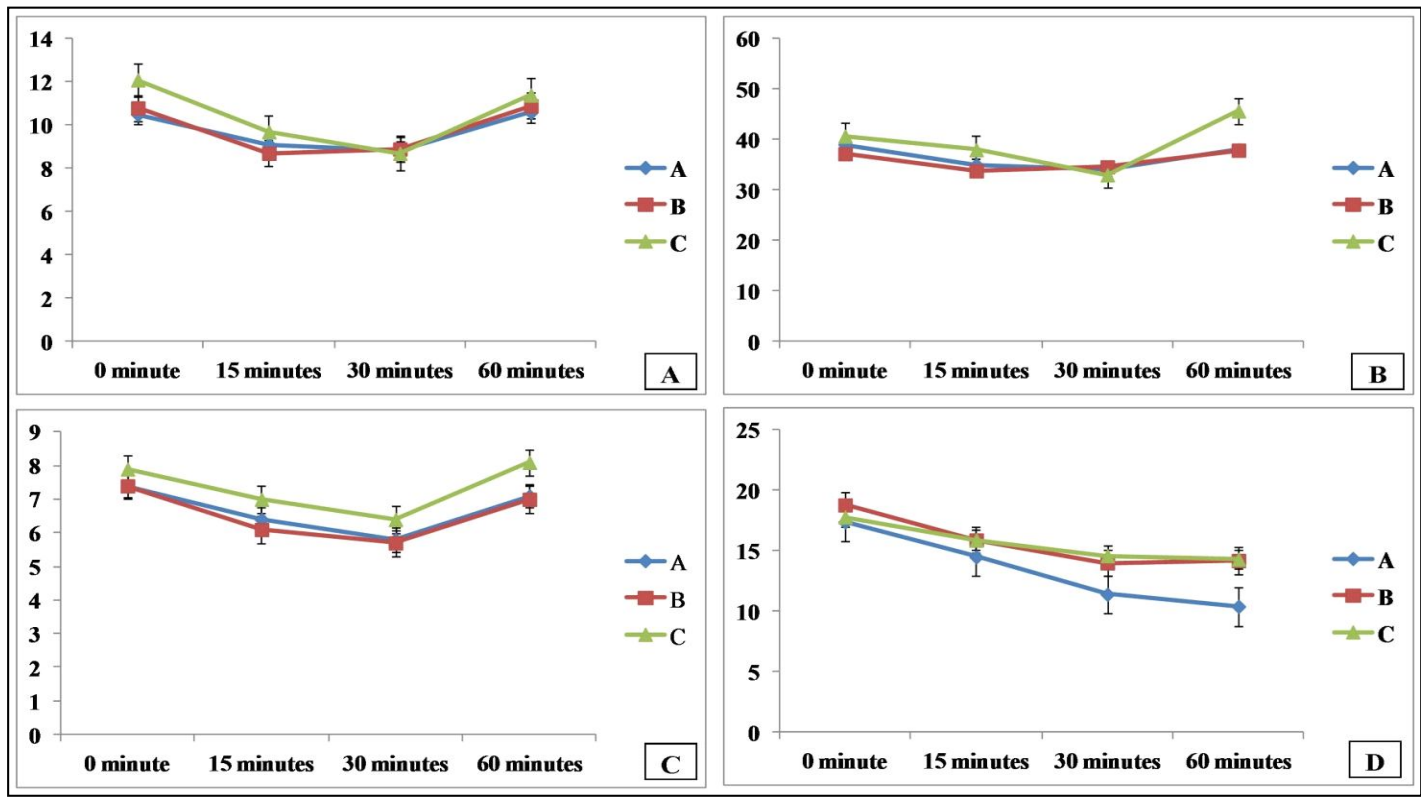

Fig 4: Effects of ketamine hydrochloride in combination with midazolam, dexmedetomidine and butorphanol on haematological parameters.

A. Haemoglobin; B. Packed cell volume; C. Total erythrocyte count; D. Total leukocyte count.
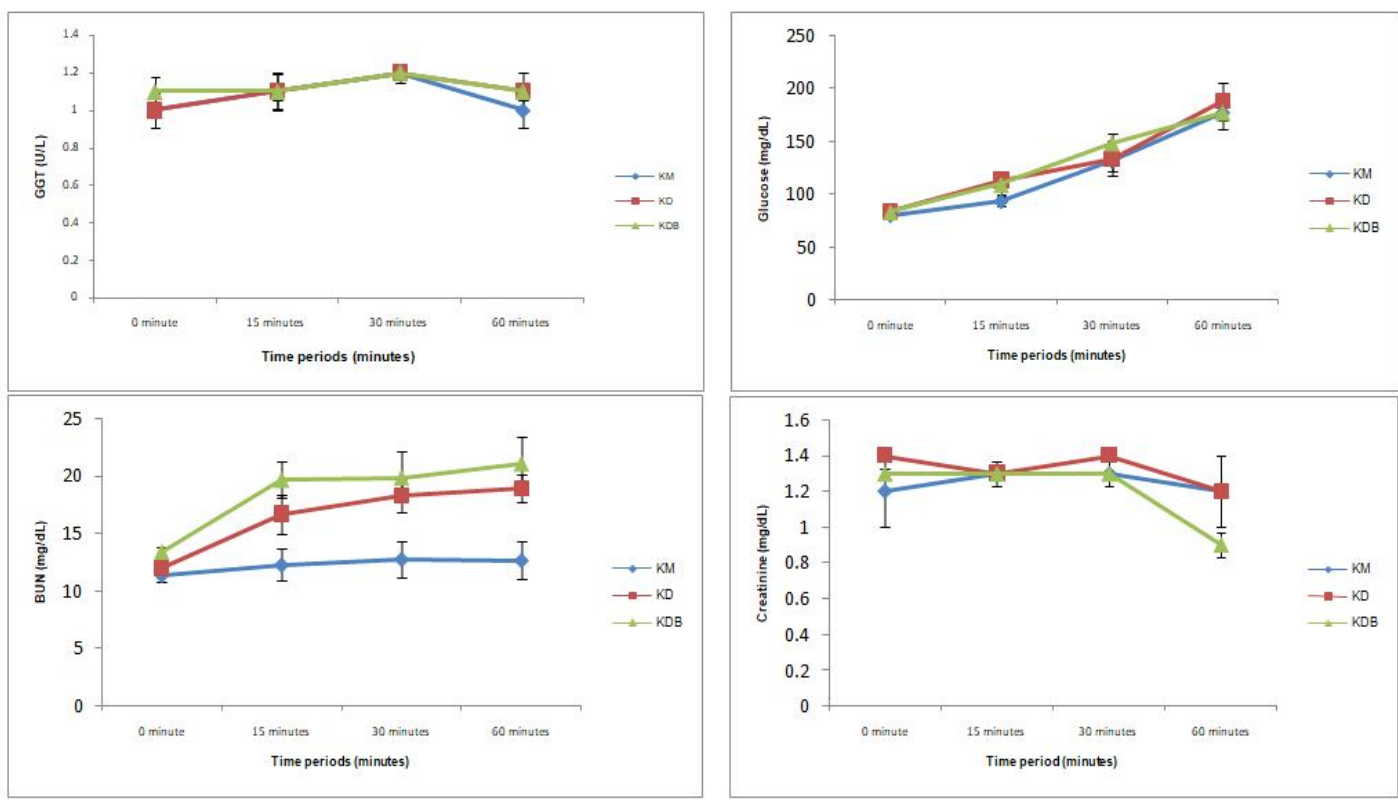

Fig 5: Effects of ketamine hydrochloride in combination with midazolam, dexmedetomidine and butorphanol on biochemical parameters. 


\section{CONCLUSION}

In conclusion, this study showed that the different drug combinations used have differential effects on clinicophysiological, haematological, serum biochemical profile in the surgical management of cats. These changes must be taken into consideration for choosing the anaesthetic protocol for surgical intervention in the cat. Treatment with ketamine hydrochloride in combination with dexmedetomidine and butorphanol led to acceptable sedation and mild changes in clinico-physiological, haematological, serum biochemical profile in comparison to the other two groups. Considering all aspects, ketamine hydrochloride in combination with dexmedetomidine and butorphanol, with glycopyrrolate premedication, showed the better result as far as peri and postoperative analgesia.

\section{Ethical approve}

Institutional Ethical Committee, College of Veterinary Sciences and Animal Husbandry, Central Agricultural University, Selesih, Aizawl, Mizoram (1476/GO/Re/SL/ CPCSEA, DATED 12 JUNE, 2019 Valid up to 11/06/2024).

\section{REFERENCES}

Ahmad, R.A., Kinjavdekar, P., Amarpal.,Aithal, H.P., Pawde, A.M. and Kumar, R. (2013). Potential use of dexmedetomidine for different levels of sedation, analgesia and anaesthesia in dogs. Vet. Med. 58(2): 87-95.

Amengual, M., Flaherty, D., Auckburally, A., Bell, A.M., Scott, E.M. and Pawson, P. (2013). An evaluation of anaesthetic induction in healthy dogs using rapid intravenous injection of propofol or Alfaxalone. Vet. Anaesth. Analg. 40(5): 115-123.

Ansah, O.B., Vainio, O., Hellsten, C. and Raekallio, M. (2002). Postoperative pain control in cats: Clinical trials with medetomidine and butorphanol. Vet. Surg. 31: 99-103.

Biermann, K., Hungerbühler, S., Mischke, R. and Kästner, S.B. (2012). Sedative, cardiovascular, haematologic and biochemical effects of four different drug combinations administered intramuscularly in cats. Vet. Anaesth. Analg. 39(2): 137-150.

Brondani, J.T., Luna, S.P.L. and Padovani, C.R. (2011). Refinement and initial validation of a multidimensional composite scale for use in assessing acute postoperative pain in cats. Am. J. Vet. Res. 72(2): 174-183.

David, W.P.A. (1993). Studies on Propofol as an intravenous general anaesthetic in dogs. Indian J. Vet. Surg. 14(1): 45-47.

Dyson, D.H. and Davies, R.J. (1999). Dose effect and benefits of glycopyrrolate in the treatment of bradycardia in anesthetized dogs. Can. Vet. J. 40: 327-331.

Granholm, M., McKusick, B.C., Westerholm, F.C. and Aspegren, J.C. (2006). Evaluation of the clinical efficacy and safety of dexmedetomidine or medetomidine in cats and their reversal with atipamezole. Vet. Anaesth. Analg. 33: 214-223.

Ilkiw, J.E., Sute,r, C.M., Farver, T.B., McNeal, D. and Steffey, E.P. (1996). The behaviour of healthy awake cats following intravenous and intramuscular administration of midazolam. J. Vet. Pharmacol. Ther. 19: 205-216.

Jacobson, J.D., Mcgrath, C.J., Ko, J.C. and Smith, E.P. (1994). Cardiorespiratory effects of glycopyrrolate-butorphanolxylazine combination, with and without nasal administration of oxygen in dogs. Am. J. Vet. Res. 55(6): 835-841.

Kelawala, N.H., Parsania, R.R. and Patil, D.B. (1991). Haematological and biochemical studies on ketamine, propofol and propofolketamine as general anaesthesia in diazepam premedicated goats (Capra hircus). Ind. J. Vet. Surg. 12(1): 17-20.

Kohrs, R. and Durieux, M.E. (1998). Ketamine: Teaching an old drug new tricks. Anesth. Analg. 87: 1186-1193.

Maddern, K., Adams, V.J., Hill, N.A. and Leece, E.A. (2010). Alfaxalone induction dose following administration of medetomidine and butorphanol in the dog. Vet. Anaesth. Analg. 37(1): 7-13.

Mantz, J., Josserand, J. and Hamada, S. (2011). Dexmedetomidine: New insights. European J. Anaesth. E.J.A. 28(1): 3-6.

Moffat, K. (2008). Addressing canine and feline aggression in the veterinary clinic. Vet. Clin. North Am. Small Anim. Pract. 38: 983-1003.

Olkkola, K.T. and Ahonen, J. (2008). Midazolam and other benzodiazepines. Modern Anesthetics. 335-360.

Pasin, L., Febres, D., Testa, V., Frati, E., Borghi, G., Landoni, G. and Zangrillo, A. (2015). Dexmedetomidine vs midazolam as preanesthetic medication in children: A meta analysis of randomized controlled trials. Pediatric Anesth. 25(5): 468-476.

Roy, B.K. (2008). Veterinary Pharmacology and Toxicology. $3^{\text {rd }}$ (edn). New Delhi, Kalayani Publishers, pp. 146-159.

Selmi, A.L., Mendes, G.M., Lins, B.T., Figueiredo, J.P. and BarbudoSelmi, G.R. (2003). Evaluation of the sedative and cardiorespiratory effects of dexmedetomidine, dexmedetomidinebutorphanol and dexmedetomidine-ketamine in cats. J. Am. Vet. Med. Assoc. 222(1): 37-41.

Shah, M., Yates, D., Hunt, J. and Murrell, J. (2019). Comparison between methadone and buprenorphine within the QUAD protocol for perioperative analgesia in cats undergoing ovariohysterectomy. J. Feline Med. Surg. 21(8): 723-731.

Shekhawat Ali, (2018). Evaluation of tramadol and meloxicam in pain management of canine ovariohysterectomy. MVSc, Thesis. LUVAS, Hisar.

Shinde, P.R., Chepte, S.D., Thorat, M.G., Raulkar, R.V., Ali, S.S., Fani, F.A., Anam, A.D., Bhave, N.P. and Vaidya, S.R. (2018). Clinical efficacy of ketofol and propofol in dog. Int. J. Sci. Environ. Technol. 7(6): 1949-1953. 